Ursprung von der Substanz von Polypen oder Zoophyten haben, welche von dieser Schwalbenart gesammelt wird ${ }^{*}$ ).

\title{
Beiträge zur botanischen Geographie des südlichen Europas;
}

rom

Professor Link.

(Aus Wiegmann's Archiv 1836 und Jameson's The Edinb. new philos. Journ. XXII. 304.)

Die Flora einer Gegend ist eine ihrer unterscheidendsten Bildungen, sie bestimmt ihren Charakter. Es ist nicht leicht, Pllanzen zu finden, die eine Gegend in Bezug auf ihre Breite sowohl, als auf ihre Länge und Höhe characterisiren. Wir müssen dazı Pflanzen auswähleu, die ausgedehnt verbreilet sind, die sich nicht leicht durch Samen fortpflanzen, weil diese leicht durch Zufall von einer Gegend einer andern zugefiihrt werden, und die nicht unter. Getraide wachsen. So fand ich in Portugal die" schöne blaue KornWume (Centaurea. Cyanus), die unsere nördlichen Felder schrnückt. Aber selbst wenn wir eine gule Auswahl getroffer haben, müssen wir nock lange in einer Gegend uns aufhalten, um die Gränzen der Verbreitung einer Pflanze zu bestimmen.

Es ist bekannt, dass viele, nicht alle, Pflanzen der nördlichen Ebene auf den Bergen. des Südens vorkommen; und obgleich solche Pflanzen sehr geeignet sind, die klimatischen Verhältnisse der Berge zu bezeichnen, so ist

*) Jaeger de Holothuriis. Turici 1833. Diese Thiere, die so sehr gesucht werden zum Luxus der Chinesen, werden an den Küsten von Sumatra, Neuguinea und Neuholland gefisclit. 
es doch nicht so für die Bestinmung der Verbältnisse des Climas der Ebene, von der wir aufsteigen. Diese Pflanzen steigen auch den Berg allmälig an; und machen nicht die wunderbaren Sprünge wie der gemeine Sanddorn (Hippophäe Rhamnoides), welchen der Reisende von Rügen bis Genf nur bis zu diesen beiden Punkten antrifft. Es ist gut, wenn die charakteristische Pflanze allgemein bekannt ist, so dass eine bedeutende botanische Kenntniss nicht nöthig ist, um die gewünschte Bestimmung zu machen. Eine Pllanze, die sehr : geeignet ist für die Bestimmung der Erhebung des Bodens, ist die Heidelbeere (Vaccinium myrtillus). Sie wächst im nördlichen.Teutschland, auch bei Berlin; in den beholzten Theilen der Ebene; sie steigt dann-allmälig auf, wir finden sie bei Freyburg in Baden, aber nur auf höberen Bergen, in der Schweiz wächst sie in den Wäldern der untern Alpen, erscheint dann zuerst wieder auf der hohen Alpe di Caporaghleno bei Livizzano, wo sie auf den Wiesen mit Colchicum autumnale vorkümmt, weiter trifft man sie nur auf dem hohen Mazella in den Abruzzen.

Kehren wir auf die Ebene zurïck und betrachten dar. nach die Gränzlinien der Pflanzen des südlichen Europa. Nachdem wir die Alpen hinter uns haben, lässt sich bald eine allgemein bekannte Pflanze; der Lavendel (Lavandula Spica) blicken. Wir treffen diese Pflanze auf den sonnigen Hügeln um Veroria, bei Coni, gegen den Col di Tenda, stets der Bergkette folgend, dann delnt sie sich nach dem Süden von Frankreich und Spanien aus, in Arragonien ist sie häufig, weiter aber im Innern und in den Ebenen won Castilien finden wir sie nicht mehr, auch nicht mehr in Portugal. Sie hört auch auf in der Ríchtung nach Rom, und erscheint nur noch zwischen den hohen Bergen der adriatischen Küste. Der Lavendel ist keine östliche. Pflanze ; in Istrien finden wir an ihrer Stelle den Salbey (Salvia offi- 
cinalis, welche dem Monte Maggiore folgt, aber in Italien nur auf den hohen Bergen in den Abruzzen.

Die Ebene der Lombardei ist ein Garten, wo wir auch kaum eine wildwachsende Pflanzenproduktion bemerken, und keine, welche.als charakteristisch angesehen werden kann. Das hügelige lstrien ist auf gleiche $W$ eise mit gepflanzten Oelbäumen bedeckt, und auch nur.zwischen diesen kann man den Anfarfg der Myrthen-Region entdecken. Die Myrthe bedeckt ganze Distrikte in Portugal. Sie ist üher den mittlern und südlichen. Theil von Spanien, die südlichen Provinzen von Frankreich verbreitet und dehnt sich bis nach Genua aus. Sie findet sich überall im Kirchenstaate und um Neapel, durch ganz Istrien bis.zum Fusse des Monte Maggiore. Sie dehnt sich etwas weiter nach Süden aus, wird aber seltener und seltener, im nördlichen Italien wächst sie nur einzeln.

Gehen wir von der Region der Myrthen in einer südlichen Richtung weiter, so kommen wir an das Land des Rosmarins oder besser des Oleanders. Dieser Strauch fängt bei Merida in. Spanien an, folgt dem Laufe der Guadiana und Ayamonte. Er schmückt dann die Thäler von Algarbien mit seinen schönen Blüthen; wälrend in der Serra de Monchique sich die schönen Blumen von Khadendron ponticum ausbreiten. Wir finden ihn nur in den warmen Thälern von Calabrien und Sicilien. In Morea, wenn man mehre Stunden den Weg nach dem alten Trözene verfolgt, über trockne dürre Gebirge, wo kleine wilde Kirschenbäume (Pyrus, cuneifolia) nur spärlichen Schutz. gegen die Sonnenstrablen gewähren, sieht man in der Ferne einen langen Streifen von Oleander, der sich mit, einigen Sycamoren um die Berge windet, dem müden Wanderer ein willkommener Anblick, denn er ist sicher, hier einen Bach zu finden, und die Sycamoren versprechen einen angenehmen: Schatten. 
Wir haben nun die drei botanischen Regionen des südlichen Europa, von Norden nach Sïden angezeigt: wir. wollen hierauf die Grenzlinien der Vegetation ron Westen nach Osten betrachten. Es ist nicht schwer, hier Pflanzen zu finden, welche die Gränzen bezeichnen, denn die Tannen und Eichen sind sehr charakteristisch.

Unsere Thiergarten Tanne (Pinus syluestris), ich will sie so nennèn, wegen der zahlreichen botanischen $Z$ weifel der Namen, steigt nicht über den Kamm der Alpen nach Süden, nicht über den Rhein nach Westen, nämlich im wilden Zustande. Es ist historisch bewiesen, dass sie oft genug in Frankreich angepflanzt wurde. Pinus pinaster Lam. (P. maritima DeC.) bildet den grossen Wald von Leiria, den.Don Diniz anplanzen liess, aber ron wilden Samen. Dieser Baum hat einen ganz verschiedenen Wachsthum angenommen, er bildet eine Pyramide, statt einer Krone; die $Z$ weige bilden fást rechte Winkel mit dem Slamm und die nadelförmigen. Blälter sind sehr lang und dunkelgrün. Er erstreckt. sich durch ganz Spanien, das südliche Frankreich, in der Nähe des mittelländischén Meers, bis er Genua erreicht. Der Pinaster hört aber bald auf und es erscheint an seiner Stelle die Aleppische Fichte (Pinus halepensis). Die langen und feinen nadelförmigen. Blälter machen diesen hohen und pittoresken Baum hinreichend bekannt. Er $\mathbf{r}_{\mathfrak{l}}$ gehört den Ebenen Italiens, während eine andere schüne Art den Bergen angehört, die uns noch nicht lange bekannt ist. Es ist der Laricio (Pinus Laricio), welcher auf den Bergen Korsikas und Calabriens und an Aetna wächst und das Ansehn und die Höhe der Rothtanne (Pinzus excelsa) hat. Er blühele sonst auf den niedrigen Bergen Italiens, wie bei Genua ; denn Strabo führt an, dass das Holz zu Masten: gefällt: und von den Einwohnern ausgefübrt wurde,, die dafïr Oel und andere Artikel eintauschten. Der Fall ist nun 
ganz umgekehrt. Vor einigen Jahren, als der. Grund des Theaters von Carlos zu Genua gelegt wurde, fand man einige Tannenzapfen, die mir nachher von Viviani gezeigt wurden, und sie waren ganz den Zapfen ähnlich, die ich vom Aetna mitgebracht hatte.

Weiter gegen Osten finden wir die. griechische Fichte (Pinus maritima), einen Baum von unbeträchllicher Hölhe, der aber eine schöne Krone hat, mit hellgrünen Nadeln beselzt, die durch ihre eigenthümliche Farbe diese Art sogleich von allen andern unterscheiden lassen. Ich fand diese Art im wilden Zustande in keiner andern Gegend als in Griechenland, und besonders im alten Attika. Bei Eleusis, am Hymeltus und auf dem Vorgebirge von Sunium sieht man Wälder dieses schönen Baumes. Wenn man die hügelichte Ebene von Megara verlaśsen, steigt der. Pfad plötzlich gegen den Isthmus an, man erreicht einen. Wald dieses Nadelholzes, Felsen erscheinen, und die Gebirge, um welche sich der Pfad windet, werden luftiger und steiler.: Der- schmale Arm der See ist fast ganz verschlossen durch die nun verlassene Insel Salamis. Man kömmt über einen gefährlichen, über das Meer herüberhängenden Felsen. Hier war es, wo im entfernten Alterthum der Räuber Pit yokam pos seine Schlachtopfer zwischen zwei Fichten band und grauenvoll ermordete. Dies konnte er leicht mit der kleinen. griechischen Fichte, aber mit unsern Arten wäre es unmöglich gewesen.

In Morea ist der Baum nicht häufig, und ist fast auf die nördliche Küste beschränkt. Er schmückt die Thäler von Epidaurus und die Berge von Aegina. Am Fusse des hohen Cyllene, gegen das Meer hin, findet man diesen Baum in seiner Vollkommenheit, und mit einer schönen und weit verbreiteten Krone wächst er an den Ufern des Xylocastro, velcher nit Heftigkeit von dem Gebirge stürzt. An der 
südlichen Küste von Morea ist er selten, und an der westlichen finden wir Pinus halepensis.

Die hier-aufgezählten drei Tannen, der Pinaster, die Aleppische Tanne und die griechische Seetanne, characterisiren drei Regionen des südlichen Europa von Osten nach Westen. Auf gleiche Weise haben wir drei Eichen. In Spanien und Portugal giebt es eine Eichenart.mit essbaren Früchten, die den Alten wohl bekannt war: Desfon tá ines entdeckte sie auf den Bergen von Algier und nannie sie Quercus Ballota. Von Hoffmannsegg und ich fanden sie in Portugal und Spanien; wegen ihrer Früchte wird sie als Waldbaum bei Portalegke in Portugal gebaut. In Italien wächst eine andere Art mit essbaren Früchten, und die Tenore merkwürdig genug als eine Varietät unserer Eiche (Quercus pedunculata) betrachtet. Enlich haben wir in Griechenland Quercus Aegilops, die hohe, schlanke und schöne Veltaridia, die arkadische Eiche, deren Frucht von den

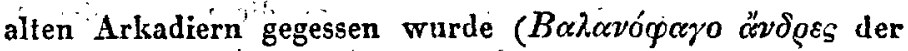
Pythia) und wovon die bei ins in der Gerberei gebraucht werdenden Knoppern kommen. Die Eiche, welche die Galläpfel liefert (Quercus infectoriá) kömmt an der östlichien Küste von Griechealand vor; häufig aber erst in Natolien.

\section{Bananen.}

Die Früchte der Banänen (Musa paradisiaca) bestehen nach Boussingnault aus: Zucker, Gummi, "Apfel -, Gallus - und Gallertsäure, thierisch vegetabilische Materie, die in der Hilze coagulirt, und Faser.

Der Saft des Bananenbaums ist durchsichtig wie Wasser und setzt an der Luft rosenfarbige Flócken ab. Er enthält : Tannin, Gallussäure, Essigsäure, Chlornatrium, Kalk -, Kali - und Thonerdensalze, 